\title{
Composición química de los componentes del cultivo de avena (Avena sativa L.), variedad Chihuahua
}

\section{Chemical composition of oat (Avena sativa L.) crop components, Chihuahua variety}

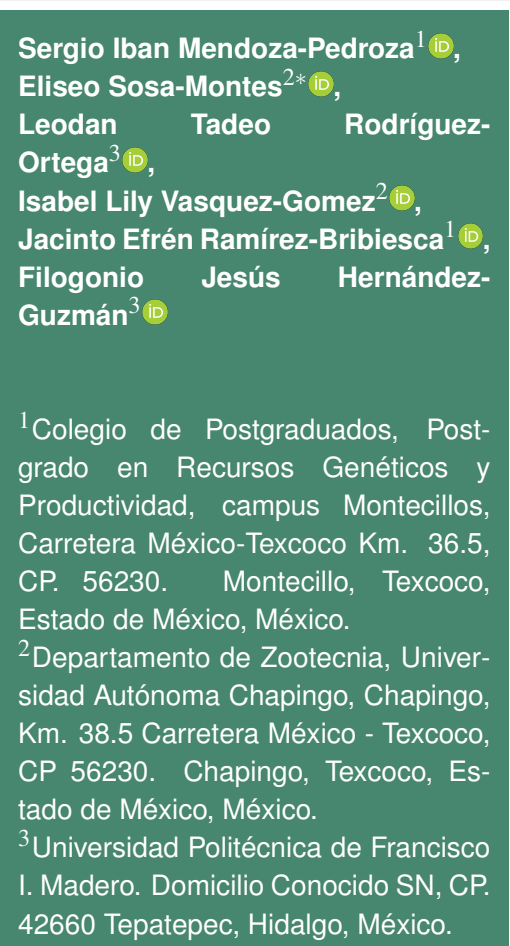

*Autor de correspondencia: eliseososa@yahoo.com.mx

Artículo científico

Recibido: 04 de marzo 2021 Aceptado: 13 de septiembre 2021

Como citar: Mendoza-Pedroza SI, Sosa-Montes E, Rodríguez-Ortega LT, Vasquez-Gomez IL, RamírezBribiesca JE, Hernández-Guzmán FJ (2021) Composición química de los componentes del cultivo de avena (Avena sativa L.), variedad Chihuahua. Ecosistemas y Recursos Agropecuarios Núm. Esp. II: e2966. DOI: 10.19136/era.a8nll.2966
RESUMEN. Por su importancia como cultivo estratégico de invierno parcialmente caracterizado, se estudió la composición química de avena forrajera variedad Chihuahua. En hoja, tallo, maleza, panícula y planta completa, se determinaron ( $\mathrm{g}$ $100 \mathrm{~g}^{-1}$ ) fibra detergente neutro (FDN), fibra detergente ácido (FDA), lignina cruda (LC), proteína cruda (PC) y grasa cruda (GC), y sus correspondientes rendimientos ( $\mathrm{kg} \mathrm{ha}^{-1}$ ) cada 15 días desde los 45 hasta los 150 días de edad (fechas de corte). La PC disminuyó $(p<0.05)$ y los contenidos de FDN, FDA y LC aumentaron $(p<0.05)$ en hoja, tallo, maleza y planta completa al avanzar la edad, por otra parte, el contenido de GC aumentó en hoja y panícula $(p<0.05)$, pero no se observaron cambios en maleza ( $p>0.05$ ). En panícula, de los 90 a los 150 días

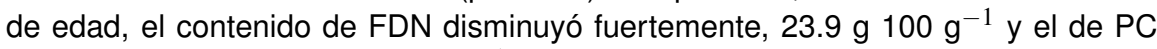

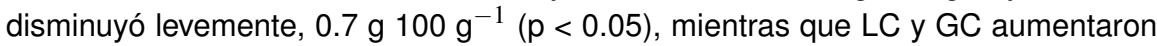
moderadamente $(p<0.05)$. Se detectó un máximo rendimiento de $P C$ en hoja y maleza a los 75 días, mientras que, en tallo el rendimiento máximo de PC se produjo a los 90 días de edad. Para que el forraje de avena, variedad Chihuahua, no contenga tanta fibra y a fin de que los contenidos de proteína y grasa, y el rendimiento de materia seca no sean tan bajos, se sugiere cosechar esta planta entre 75 y 90 días de edad.

Palabras clave: Avena sativa L., proteína cruda, fibra detergente neutro.

ABSTRACT. For its importance as a partially characterized strategic winter crop, the chemical composition of forage oat Chihuahua variety was studied. In leaf, stem, weed, panicle and whole plant, neutral detergent fiber (NDF), acid detergent fiber (ADF), crude lignin (CL), crude protein (CP) and crude fat (CF) were determined ( $\mathrm{g} 100 \mathrm{~g}^{-1}$ ), and their corresponding yields $\left(\mathrm{kg} \mathrm{ha}^{-1}\right)$ were evaluated every 15 days from 45 to 150 days of age (cutting dates). The CP in leaf, stem, weed and whole plant decreased $(p<0.05)$ while NDF, ADF and CL contents increased $(p<0.05)$ as age progressed, on the other hand, the CF content increased with age in leaf and panicle $(p<0.05)$, but no changes were observed in weed $(p>0.05)$. In panicle, from 90 to 150 days of age, the NDF content strongly decreased, $23.9 \mathrm{~g}$

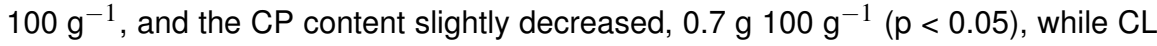
and CF increased moderately $(p<0.05)$. At 75 days of age, a maximum yield of CP was detected in leaf and weed, while the maximum CP yield in stem was produced at 90 days of age. For the oat forage, Chihuahua variety does not contain so much fiber and in order that protein and fat contents, and dry matter yield are not so low, it is suggested to harvest this plant between 75 and 90 days of age.

Key words: Avena sativa L., crude protein, neutral detergent fiber. 


\section{INTRODUCCIÓN}

La ganadería es una actividad del sector primario con fines de producción para el consumo humano. Es muy antigua y forma una parte importante de la economía de México, $11^{\circ}$ lugar como productor mundial de ganadería primaria (SIAP 2018). Alrededor de 109.8 millones de hectáreas, es decir, cerca del $56 \%$ de la superficie total de México son grandes áreas donde se desarrollan principalmente las ganaderías bovina, porcina, ovina, caprina y aviar. Diferentes factores dentro de los sistemas de producción animal afectan al ganado. Uno de ellos son los forrajes que se utilizan para su alimentación (SIAP 2018). Los forrajes son muy importantes por ser la fuente más económica de nutrimentos para los rumiantes, y constituyen aproximadamente el $80 \%$ del alimento consumido durante su vida productiva (González 1993).

Uno de los grandes problemas que enfrentan los productores en la época de invierno, es la escasez de forrajes. Los cereales forrajeros son indispensables en época de invierno (Hernández-Garay y Martínez 1997). El cultivo de avena (Avena sativa L.) tiene gran importancia en México para la producción de alimento balanceado de uso pecuario y por su amplio rango de adaptación, tanto a zonas altas, frías y lluviosas, como a ambientes semiáridos, es un cultivo estratégico. La superficie cosechada de avena forrajera en México fue de 124741 ha, con producción de $3103472 \mathrm{t}$ y rendimiento promedio de $24.9 \mathrm{t} \mathrm{ha}^{-1}$ (SIAP 2018).

En los últimos años en México, ha aumentado el número de variedades de avena, de 1960 a la fecha el Instituto Nacional de Investigaciones Forestales, Agrícolas y Pecuarias (INIFAP) ha sido la principal estación donde se han liberado variedades de avena (Leyva-Mir et al. 2018). En su estudio, estos autores mencionan 28 variedades de avena sembradas bajo temporal en los valles altos de México. La avena se utiliza en cualquier etapa de crecimiento para el consumo animal, desde germinados en la alimentación de especies menores, hasta en estado de madurez fisiológica (Ortiz-Tirado et al. 2019, LeyvaMir et al. 2018). Por esto se plantea la necesidad de evaluar distintos materiales, como la variedad Chihuahua, a efecto de identificar su mejor comportamiento. Conocimiento que contribuirá, a hacer un uso más provechoso de este recurso. Por lo anterior el objetivo de este estudio fue determinar la composición química del forraje de Avena sativa variedad Chihuahua a diferentes edades de corte para conocer el punto de máximo rendimiento de nutrientes.

\section{MATERIALES Y MÉTODOS}

\section{Localidad}

Las muestras se obtuvieron del Campo Experimental Predio Nuevo perteneciente al Colegio de Postgraduados, ubicado en Montecillo, Texcoco, estado de México, $19^{\circ} 21^{\prime} \mathrm{N}, 98^{\circ} 53^{\prime} \mathrm{O}$, con altitud de $2240 \mathrm{msnm}$. El clima es templado subhúmedo, el más seco de los subhúmedos, temperatura media anual $15.2{ }^{\circ} \mathrm{C}$, precipitación media anual de 636.5 $\mathrm{mm}$, lluvias en verano de junio a octubre (García 2004). A partir de los 45 días de edad se obtuvieron plantas de las parcelas experimentales, cada 15 días hasta los 150 días de edad.

\section{Obtención de las muestras}

Las plantas (45 días de edad y subsecuentes) se cortaron de forma manual, embolsaron, etiquetaron y secaron a $55{ }^{\circ} \mathrm{C}$ en estufa de aire forzado hasta peso constante. Posteriormente, se molieron y se identificaron, colocándose en bolsas de plástico para su análisis en el laboratorio de Nutrición Animal del Departamento de Zootecnia de la Universidad Autónoma Chapingo (UACH), estado de México.

\section{Componentes del cultivo, composición química, y rendimientos de nutrientes}

Las proporciones de maleza a los $45,60,75$, 90, 105, 120 y 150 días de edad fueron 9.9, 9.4, $8.8,8.3,6.2,5.3,5.4$ y $4.6 \%$, respectivamente. Las principales familias de malezas fueron Poace, Portulacaceae y Malvaceae. Seis muestras (tres parcelas con dos muestras por parcela) de cada componente se obtuvieron a los $45,60,75,90,105,120,135$ y 150 días de edad. Para determinar el contenido de nutrientes de seis repeticiones y la composición 
química con tres repeticiones. Las parcelas se establecieron en 2018, la siembra se realizó al voleo. No hubo fertilización y en ausencia de lluvias, se proporcionaron riegos a capacidad de campo cada dos semanas. Se determinó en el laboratorio las siguientes variables en $\mathrm{g} 100 \mathrm{~g}^{-1}$ en base seca: fibra detergente neutro (FDN), fibra detergente ácido (FDA), lignina cruda (LC) (Van Soest 1994), proteína cruda (PC), cenizas (Cen), grasa cruda (GC) (AOAC 1980), y a partir de estos valores y del rendimiento de materia seca para 45, 60, 75, 90, 105, 120, 135 y 150 días de edad (1921, 2042, 3649, 5030, 5339, 5693,5371 y $4877 \mathrm{~kg} \mathrm{ha}^{-1}$, respectivamente), se estimaron los rendimientos ( $\mathrm{kg} \mathrm{ha}^{-1}$ ): RFDN, RFDA, RLC, RPC, RCen y RGC. Se analizaron cinco componentes del cultivo (hoja, tallo, panícula, maleza y planta completa).

\section{Análisis estadístico}

El análisis estadístico se realizó con el paquete estadístico SPSS (Dhakre et al. 2018). El diseño estadístico fue de bloques completos al azar con tres repeticiones. El análisis de varianza se realizó a través del procedimiento del modelo general lineal. Para comparar las edades de cosecha se utilizó la prueba de Tukey $(\alpha=0.05)$.

\section{RESULTADOS}

\section{Contenidos de nutrientes}

En hoja (Tabla 1), los contenidos de FDN, FDA y LC aumentaron $26.8,18$ y $2.8 \%(p<0.05)$ respectivamente, al avanzar la fecha de corte de 45 a 150 días y los de PC y Cen disminuyeron $(p<0.05) 17.1 \%$ (de 25.8 a $8.7 \%$ ) y $2.0 \%$, respectivamente. En hoja, la FDN aumentó más que lo que disminuyó la PC, la diferencia entre estos dos cambios fue del $9.7 \%$.

En tallo (Tabla 2), los contenidos de FDN, FDA y LC aumentaron $26.6,25.9$ y $4.0 \%(p<0.05)$ respectivamente, al avanzar la fecha de corte de 45 a 150 días y los de PC y Cen disminuyeron 12.3 y $6.8 \%$ (p $<0.05$ ), respectivamente. En tallo, la FDN aumentó más que lo que disminuyó la PC, la diferencia de estos cambios fue del $19.8 \%$.
En maleza (Tabla 3), los contenidos de FDN, FDA y LC aumentaron 24.9, 32.4 y $4.6 \%(p<0.05)$ respectivamente, al avanzar la fecha de corte de 45 a 150 días y los de PC y Cen disminuyó 20.7 y $7.8 \%$ $(p<0.05)$, respectivamente. En maleza (Tabla 3), la FDN aumentó más que lo que disminuyó la PC, la diferencia de estos cambios fue del $4.2 \%$, valor positivo menor que el del tallo.

Contrario a los demás componentes del cultivo, en panícula (Tabla 4), los contenidos de FDN, FDA y PC disminuyeron $(p<0.05) 23.9,2.8$ y $1 \%$, al avanzar la fecha de corte de 90 a 150 días. En panícula, tanto la FDN como la PC disminuyeron, la diferencia de estos cambios fue de $-22.9 \%$, valor negativo. Es decir, la panícula tiene un comportamiento muy distinto a los demás componentes morfológicos. Disminuyendo la FDN de forma fuerte, mientras que la PC disminuye muy poco, por lo que su diferencia es fuertemente negativa.

En planta completa (Tabla 5), los contenidos de FDN, FDA y LC aumentaron 24.5, 20.0 y $2.6 \%$ ( $p$ $<0.05$ ) al avanzar la fecha de corte de 45 a 150 días, y los de PC y Cen disminuyeron 13.7 y $4.9 \%$ ( $p<$ $0.05)$, respectivamente. En planta completa como en hoja, tallo y maleza, el aumento de FDN fue mayor que la disminución de PC, la diferencia de estos cambios fue de $10.8 \%$, valor positivo, parecido al de hoja. La planta completa de avena Chihuahua cosechada a los 150 días de edad mostró valores de PC bajos (6.8 g $100 \mathrm{~g}^{-1}$ ). Esta disminución de PC fue de $13.7 \%$ al pasar de 45 a 150 días de edad (20.5 - 6.8\%).

El contenido de GC aumentó $(p<0.05)$ hasta los 120 días de edad en todos los componentes del cultivo, llegando a ser en ellos $2.3 \%$ en promedio a los 120 días, mientras que, en panícula, la GC siguió aumentando hasta los 150 días, fecha en que fue $6 \%$. Por lo que los contenidos de FDN, FDA y GC en hoja, tallo, maleza y planta completa aumentaron al avanzar la edad y los de PC disminuyeron. En panícula tanto FDN como PC disminuyeron, pero la PC lo hizo levemente y la FDN disminuyó de forma fuerte. Adicionalmente la GC aumentó fuertemente en la panícula y levemente en los demás componentes del cultivo. 
Tabla 1. Composición química de la hoja de avena ( $100 \mathrm{~g}^{-1}$, base seca) en función de la fecha de corte.

\begin{tabular}{ccccccc}
\hline Fecha de corte, días & FDN & FDA & LC & PC & Cen & GC \\
\hline 45 & $41.1^{f}$ & $25.0^{g}$ & $1.7^{f}$ & $25.8^{a}$ & $11.2^{a}$ & $2.4^{e}$ \\
60 & $43.2^{e}$ & $25.8^{f}$ & $1.9^{e f}$ & $25.5^{a}$ & $11.1^{a}$ & $2.3^{e}$ \\
75 & $52.5^{d}$ & $30.4^{d e}$ & $2.2^{d}$ & $20.8^{b}$ & $9.9^{c}$ & $2.6^{d}$ \\
90 & $52.2^{d}$ & $30.8^{d}$ & $2.1^{d e}$ & $20.1^{b}$ & $10.3 b^{c}$ & $2.6^{d}$ \\
105 & $54.0^{c}$ & $30.0^{e}$ & $2.6^{c}$ & $18.8^{c}$ & $9.9^{c}$ & $3.2^{c}$ \\
120 & $59.8^{b}$ & $32.5^{c}$ & $3.2^{b}$ & $12.9^{d}$ & $10.5^{b}$ & $3.9^{a}$ \\
135 & $67.4^{a}$ & $41.3^{b}$ & $4.8^{a}$ & $8.3^{e}$ & $10.1^{b c}$ & $3.7^{b}$ \\
150 & $67.9^{a}$ & $43.0^{a}$ & $4.5^{a}$ & $8.7^{e}$ & $9.2^{d}$ & $3.1^{c}$ \\
EE & 0.20 & 0.23 & 0.10 & 0.22 & 0.12 & 0.06 \\
\hline
\end{tabular}

Superíndices distintos en una misma columna indican diferencias estadísticas
(Tukey, $\alpha=0.05$ ). FDN: fibra detergente neutro, FDA: Fibra detergente ácido, LC: lignina cruda, PC: proteína cruda, Cen: cenizas, GC: grasa cruda. EE: error estándar.

Tabla 2. Composición química del tallo de avena ( $100 \mathrm{~g}^{-1}$, base seca) en función de la fecha de corte.

\begin{tabular}{ccccccc}
\hline Fecha de corte, días & FDN & FDA & LC & PC & Cen & GC \\
\hline 45 & $53.9^{g}$ & $30.7^{g}$ & $1.8^{f}$ & $16.0^{a}$ & $12.7^{b}$ & $2.1^{e}$ \\
60 & $57.3^{f}$ & $32.3^{f}$ & $1.8^{e f}$ & $15.2^{a}$ & $13.4^{a}$ & $1.2^{e}$ \\
75 & $66.5^{e}$ & $39.2^{e}$ & $1.6^{d}$ & $10.4^{b}$ & $9.9^{c}$ & $1.1^{d}$ \\
90 & $71.7^{d}$ & $46.1^{d}$ & $2.3^{d e}$ & $7.2^{b}$ & $8.2^{d}$ & $1.7^{d}$ \\
105 & $72.7^{c}$ & $49.4^{c}$ & $3.0^{c}$ & $5.7^{c}$ & $6.5^{e}$ & $1.9^{c}$ \\
120 & $75.2^{b}$ & $48.5^{c}$ & $3.6^{b}$ & $5.1^{d}$ & $6.4^{e}$ & $1.9^{a}$ \\
135 & $80.2^{a}$ & $53.5^{b}$ & $3.7^{a}$ & $4.4^{e}$ & $5.9^{f}$ & $1.1^{b}$ \\
150 & $80.5^{a}$ & $56.6^{a}$ & $5.8^{a}$ & $3.7^{e}$ & $5.9^{f}$ & $1.8^{c}$ \\
EE & 0.19 & 0.54 & 0.28 & 0.25 & 0.10 & 0.07 \\
\hline
\end{tabular}

Superíndices distintos en una misma columna indican diferencias estadísticas (Tukey, $\alpha=0.05)$. FDN: fibra detergente neutro, FDA: Fibra detergente ácido, LC: lignina cruda, PC: proteína cruda, Cen: cenizas, GC: grasa cruda. EE: error estándar

Tabla 3. Composición química de la maleza asociada al cultivo de avena ( $g 100$ $\mathrm{g}^{-1}$, base seca) en función de la fecha de corte.

\begin{tabular}{ccccccc}
\hline Fecha de corte, días & FDN & FDA & LC & PC & Cen & GC \\
\hline 45 & $43.9^{g}$ & $25.8^{f}$ & $3.5^{e}$ & $27.4^{a}$ & $16.1^{b}$ & $1.5^{c}$ \\
60 & $38.6^{h}$ & $26.4^{f}$ & $3.9^{e}$ & $25.1^{b}$ & $18.1^{a}$ & $2.1^{b}$ \\
75 & $49.4^{f}$ & $35.4^{e}$ & $6.1^{d}$ & $16.7^{c}$ & $13.8^{c}$ & $1.1^{c}$ \\
90 & $56.2^{e}$ & $39.1^{d}$ & $8.4^{a}$ & $14.7^{d}$ & $12.8^{d}$ & $1.9^{b}$ \\
105 & $57.4^{d}$ & $41.6^{c}$ & $7.5^{c}$ & $11.2^{f}$ & $10.6^{e}$ & $0.5^{d}$ \\
120 & $60.7^{c}$ & $45.1^{b}$ & $7.9^{b c}$ & $12.6^{e}$ & $10.3^{e}$ & $2.7^{a}$ \\
135 & $67.6^{b}$ & $45.4^{b}$ & $8.1^{a b}$ & $9.5^{g}$ & $9.8^{e}$ & $2.1^{b}$ \\
150 & $68.8^{a}$ & $58.2^{a}$ & $8.1^{a b}$ & $6.7^{h}$ & $8.3^{f}$ & $1.1^{c}$ \\
EE & 0.33 & 0.29 & 0.15 & 0.14 & 0.28 & 0.11
\end{tabular}

Superíndices distintos en una misma columna indican diferencias estadísticas (Tukey, $\alpha=0.05$ ). FDN: fibra detergente neutro, FDA: Fibra detergente ácido, LC: lignina cruda, PC: proteína cruda, Cen: cenizas, GC: grasa cruda. EE: error estándar.

\section{Rendimientos de nutrientes}

En hoja y en malezas, el RPC y los demás rendimientos de nutrientes aumentaron hasta los 75 días de edad, posteriormente RPC y RCen disminuyeron. En hoja, el RPC disminuyó hasta llegar a los 150 días de corte al 10\% del rendimiento máximo (Figura 1). En tallo, los rendimientos RPC y RCen fueron máximos a los 90 días de edad y posteriormente disminuyeron (Figura 2) hasta llegar a los 150 días del corte a 54 y $75 \%$ del rendimiento máximo, respectivamente. Contrariamente, RFDN, RFDA y RGC siguieron aumentando y fueron máxi- 
Tabla 4. Composición química de la panícula de avena (g $100 \mathrm{~g}^{-1}$, base seca) en función de la fecha de corte.

\begin{tabular}{ccccccc} 
Fecha de corte, días & FDN & FDA & LC & PC & Cen & GC \\
\hline 90 & $76.6^{a}$ & $37.2^{a}$ & $1.8^{b}$ & $11.1^{a}$ & $4.6^{b}$ & $0.4^{e}$ \\
105 & $66.1^{b}$ & $37.5^{a}$ & $1.8^{b}$ & $9.5^{b}$ & $4.5^{b c}$ & $2.1^{d}$ \\
120 & $51.4^{d}$ & $35.2^{b}$ & $2.7^{a}$ & $10.1^{a b}$ & $4.3^{c d}$ & $4.7^{c}$ \\
135 & $49.6^{e}$ & $24.3^{c}$ & $2.4^{a b}$ & $10.5^{a b}$ & $4.1^{d}$ & $5.8^{b}$ \\
150 & $52.7^{c}$ & $34.4^{b}$ & $2.7^{a}$ & $10.4^{a b}$ & $4.9^{a}$ & $6.0^{a}$ \\
EE & 0.13 & 0.46 & 0.18 & 0.36 & 0.06 & 0.81
\end{tabular}

Superíndices distintos en una misma columna indican diferencias estadísticas (Tukey, $\alpha=0.05)$. FDN: fibra detergente neutro, FDA: Fibra detergente ácido, LC: lignina cruda, PC: proteína cruda, Cen: cenizas, GC: grasa cruda. EE: error estándar.

Tabla 5. Composición química de la planta completa de avena (g $100 \mathrm{~g}^{-1}$, base seca) en función de la fecha de corte.

\begin{tabular}{ccccccc}
\hline Fecha de corte, días & FDN & FDA & LC & PC & Cen & GC \\
\hline 45 & $39.7^{h}$ & $23.6^{f}$ & $1.5^{e}$ & $20.5^{a}$ & $10.4^{a}$ & $2.0^{d}$ \\
60 & $41.9^{g}$ & $24.5^{e}$ & $1.6^{e}$ & $19.5^{b}$ & $10.4^{a}$ & $1.7^{f}$ \\
75 & $51.1^{f}$ & $29.9^{d}$ & $1.8^{c d}$ & $14.7^{c}$ & $8.7^{b}$ & $1.7^{f}$ \\
90 & $59.6^{c}$ & $34.9^{c}$ & $1.9^{c d}$ & $10.6^{d}$ & $7.0^{c}$ & $1.5^{g}$ \\
105 & $55.6^{d}$ & $34.8^{c}$ & $2.1^{c}$ & $8.0^{e}$ & $5.5^{d}$ & $1.9^{e}$ \\
120 & $54.6^{e}$ & $35.1^{c}$ & $2.7^{b}$ & $6.9^{f}$ & $5.3^{e}$ & $2.4^{c}$ \\
135 & $62.4^{b}$ & $37.6^{b}$ & $3.1^{b}$ & $7.2^{f}$ & $5.4^{d e}$ & $3.6^{a}$ \\
150 & $64.2^{a}$ & $43.6^{a}$ & $4.1^{a}$ & $6.8^{f}$ & $5.5^{d}$ & $3.2^{b}$ \\
EE & 0.11 & 0.19 & 0.14 & 0.18 & 0.05 & 0.04 \\
\hline
\end{tabular}

$\overline{\text { Superíndices distintos en una misma columna indican diferencias estadísticas }}$ (Tukey, $\alpha=0.05$ ). FDN: fibra detergente neutro, FDA: Fibra detergente ácido, LC: lignina cruda, PC: proteína cruda, Cen: cenizas, GC: grasa cruda. EE: error estándar.

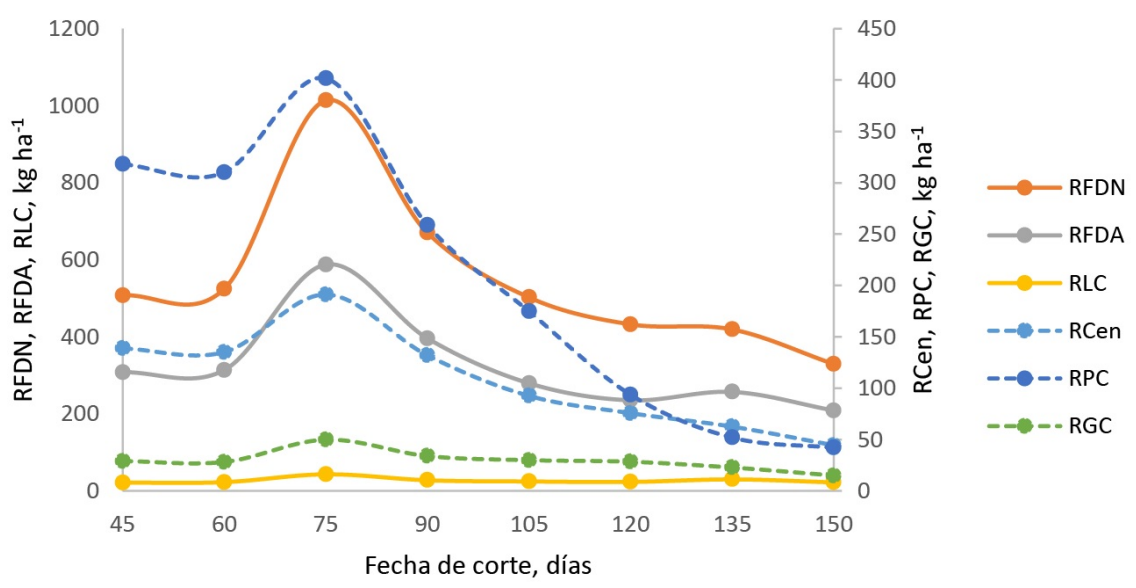

Figura 1. Rendimientos de los nutrientes de hoja de avena $\left(\mathrm{kg} \mathrm{ha}^{-1}\right)$ en función de la fecha de corte. RFDN: rendimiento de fibra detergente neutro, RFDA: rendimiento de fibra detergente ácido, RLC: rendimiento de lignina cruda, RCen: rendimiento de cenizas, RPC: rendimiento de proteína cruda, RGC: rendimiento de grasa cruda. Las variables RCen, RPC y RGC, se leen en el eje vertical derecho. Los errores estándar de la media para RFDN, RFDA, RLC, RCen, RPC y RGC fueron: $31.6,18.1,1.1,7.1,19.3$ y $1.5 \mathrm{~kg} \mathrm{ha}^{-1}$, respectivamente.

mos a los 135 días o más (Figura 3).

En maleza, los rendimientos RPC y RCen aumentaron hasta los 75 días de edad y posteriormente disminuyeron hasta llegar a los 150 días del corte a 26 y $39 \%$ del rendimiento máximo, respectivamente. Los rendimientos RFDN y RLC aumentaron hasta los 


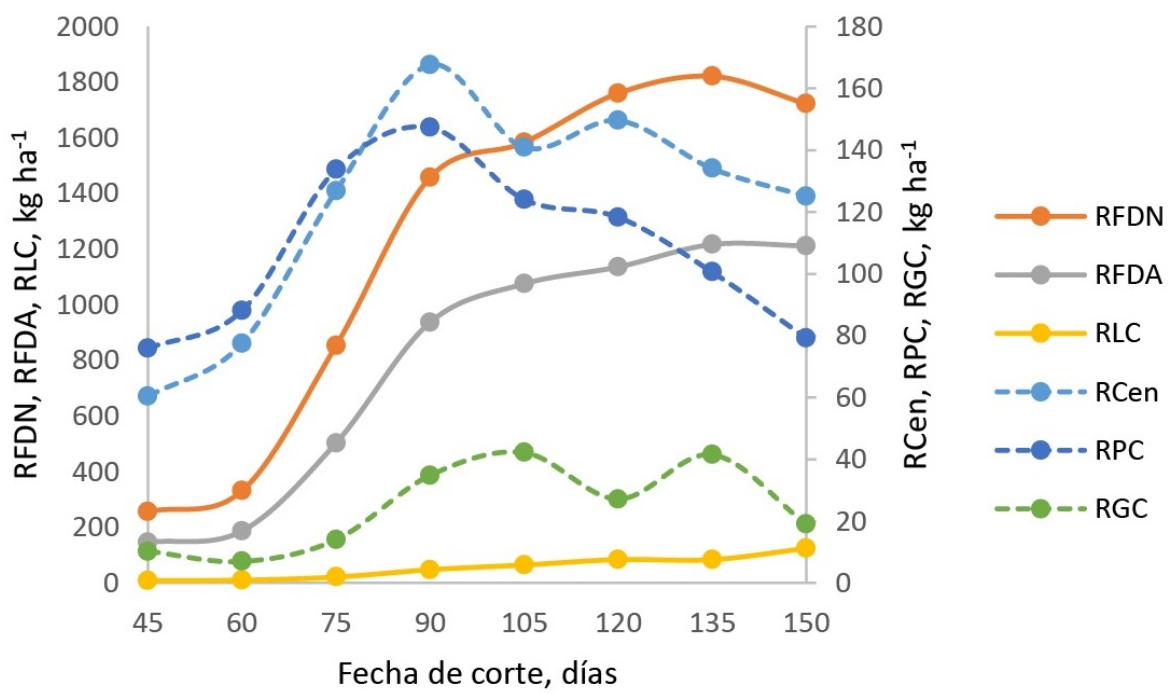

Figura 2. Rendimientos de los nutrientes del tallo de avena $\left(\mathrm{kg} \mathrm{ha}^{-1}\right)$ en función de la fecha de corte. RFDN: rendimiento de fibra detergente neutro, RFDA: rendimiento de fibra detergente ácido, RLC: rendimiento de lignina cruda, RCen: rendimiento de cenizas, RPC: rendimiento de proteína cruda, RGC: rendimiento de grasa cruda. Las variables RCen, RPC y RGC, se leen en el eje vertical derecho. Los errores estándar de la media para RFDN, RFDA, RLC, RCen, RPC y RGC fueron: 90.9, 63.4, 5.7, 5.4, 4.1 y $1.9 \mathrm{~kg} \mathrm{ha}^{-1}$, respectivamente.

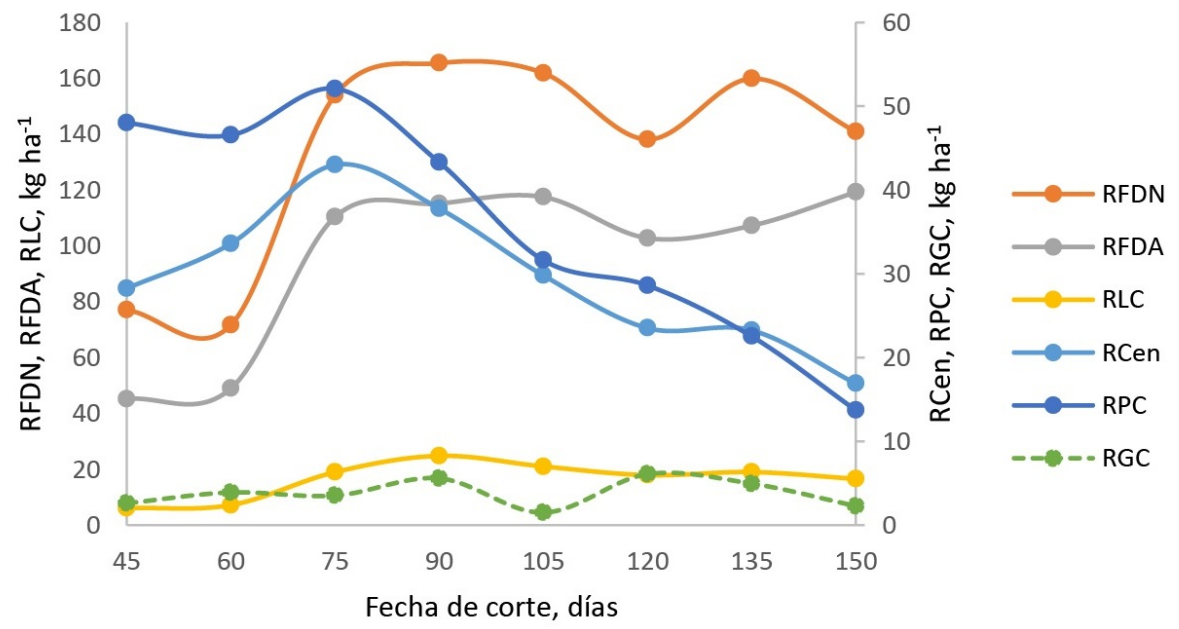

Figura 3. Rendimientos de los nutrientes de la maleza asociada con el cultivo de avena ( $\mathrm{kg} \mathrm{ha}^{-1}$ ) en función de la fecha de corte. RFDN: rendimiento de fibra detergente neutro, RFDA: rendimiento de fibra detergente ácido, RLC: rendimiento de lignina cruda, RCen: rendimiento de cenizas, RPC: rendimiento de proteína cruda, RGC: rendimiento de grasa cruda. Las variables RCen, RPC y RGC, se leen en el eje vertical derecho. Los errores estándar de la media para RFDN, RFDA, RLC, RCen, RPC y RGC fueron: $5.7,4.5,0.9,1.3,2.0$ y $0.2 \mathrm{~kg} \mathrm{ha}^{-1}$, respectivamente.

90 días, después de los cuales fueron constantes, el RGC alcanzó un máximo a los 130 días y el RFDA siguió aumentando hasta los 150 de edad (Figura 3). En panícula, el RPC y los demás rendimientos, siguieron aumentando (Figura 4) hasta los 150 días edad. No se detectaron máximos de rendimiento en este componente del cultivo.

En planta completa, los rendimientos RPC y RCen fueron máximos a los 75 días de edad y posteriormente disminuyeron (Figura 5 ), hasta llegar a 


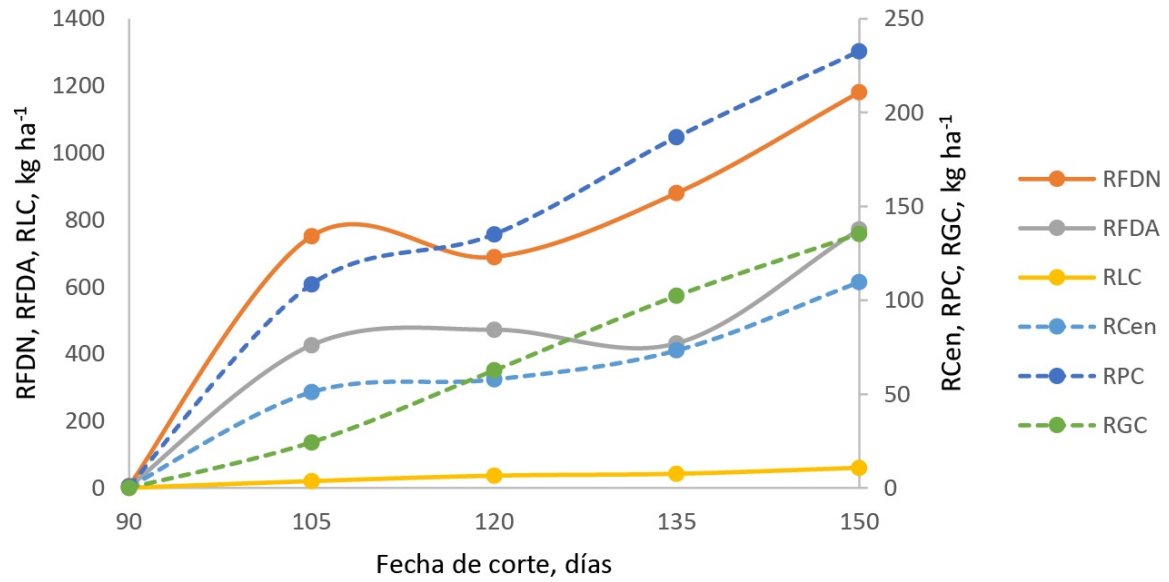

Figura 4. Rendimientos de los nutrientes de la panícula de avena $\left(\mathrm{kg} \mathrm{ha}^{-1}\right)$ en función de la fecha de corte. RFDN: rendimiento de fibra detergente neutro, RFDA: rendimiento de fibra detergente ácido, RLC: rendimiento de lignina cruda, RCen: rendimiento de cenizas, RPC: rendimiento de proteína cruda, RGC: rendimiento de grasa cruda. Las variables RCen, RPC y RGC, se leen en el eje vertical derecho. Los errores estándar de la media para RFDN, RFDA, RLC, RCen, RPC y RGC fueron: 73.3, 46.4, 3.8, 6.7, 14.9 y $9.3 \mathrm{~kg} \mathrm{ha}^{-1}$, respectivamente.

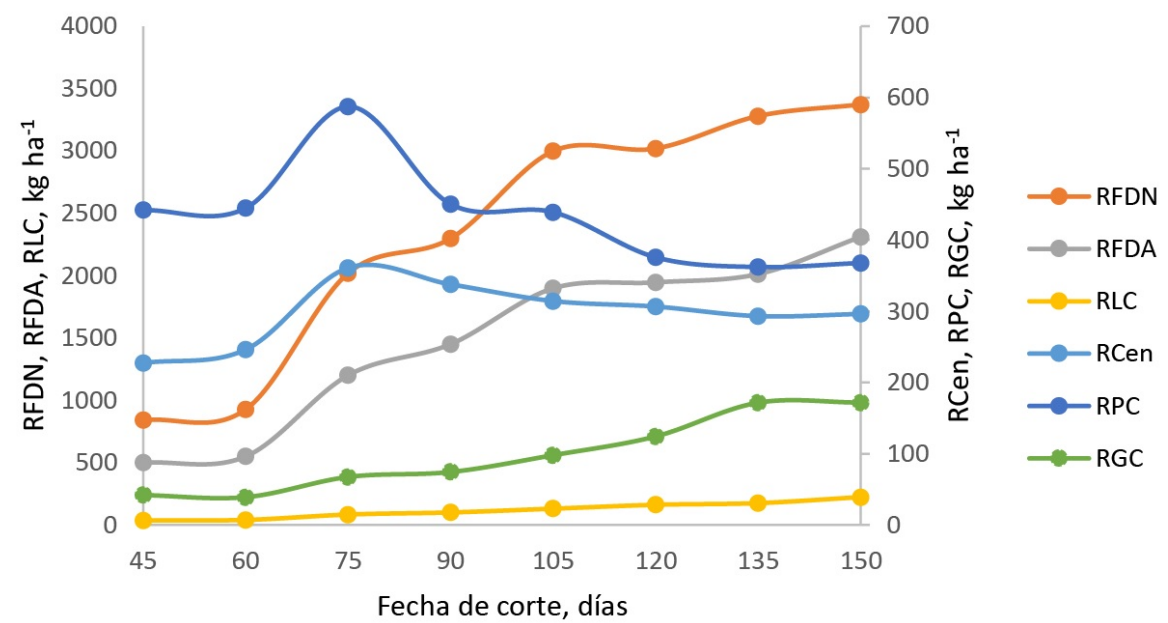

Figura 5. Rendimientos de los nutrientes de la planta completa de avena $\left(\mathrm{kg} \mathrm{ha}^{-1}\right)$ en función de la fecha de corte. RFDN: rendimiento de fibra detergente neutro, RFDA: rendimiento de fibra detergente ácido, RLC: rendimiento de lignina cruda, RCen: rendimiento de cenizas, RPC: rendimiento de proteína cruda, RGC: rendimiento de grasa cruda. Las variables RCen, RPC y RGC, se leen en el eje vertical derecho. Los errores estándar de la media para RFDN, RFDA, RLC, RCen, RPC y RGC fueron: 142.0, 95.4, 9.2, $7.9,12.9$ y $7.3 \mathrm{~kg} \mathrm{ha}^{-1}$, respectivamente.

los 150 días del corte a 63 y $82 \%$ del rendimiento máximo, respectivamente. Contrariamente, RFDN, RFDA y RLC aumentaron hasta los 150 días de edad. Estos cambios en rendimientos fueron similares a los del tallo (Figura 2). En la panícula, los cambios de RPC y RCen tuvieron un patrón similar en los demás componentes del cultivo. Este patrón consistió en al- canzar un máximo a los 75 días de edad y posteriormente disminuir a valores muy bajos. Comparando el eje vertical derecho con el izquierdo, se observa que los máximos rendimientos de nutrientes son RFDN. En el eje vertical derecho se observa que, excluyendo el tallo, a los 90 días de edad, RPC superó a RCen. 


\section{DISCUSIÓN}

\section{Porcentajes de nutrientes}

Las disminuciones de PC iniciaron entre los 75 y 90 días de edad. A los 75 días de edad, el valor de PC en planta completa (Tabla 5) fue $14.7 \mathrm{~g} 100$ $\mathrm{g}^{-1}$ (3649 $\mathrm{kg} \mathrm{ha}^{-1}$ de materia seca), y a los 90 días de edad de $10.6{\mathrm{~g} 100 \mathrm{~g}^{-1} \text { (5030 kg ha }}^{-1}$ de materia seca). Debido a esta disminución del contenido de PC, conviene cosechar entre 75 y 90 días de edad. El rendimiento máximo de materia seca fue de 5693 $\mathrm{kg} \mathrm{ha}^{-1}$, y se produjo a los 120 días de edad. Por tanto, a los 90 días de edad, el rendimiento de materia seca está cerca del máximo y el valor de PC no ha disminuido demasiado. Los resultados obtenidos son distintos a los de Espitia et al. (2012) en planta de Avena sativa genotipo Saia, quienes encontraron que

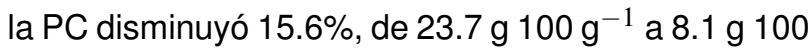
$\mathrm{g}^{-1}$ al pasar de las etapas de embuche, a grano masoso. Mientras que El-Shatnawi et al. (2004) encontraron que el valor de PC disminuyó $19.3 \%$, de 22.4

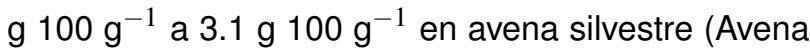
fatua) de 60 a 140 días después de la emergencia. Tal como en el presente estudio, a una mayor edad se produce una mayor disminución del contenido de proteína (El-Shatnawi et al. 2004, Espitia et al. 2012). Los contenidos de proteína del presente trabajo a los 150 días de edad, cayeron en el rango de los valores reportados por Sahu y Tiwari (2020), que variaron de 5.3 a $7.5 \mathrm{~g} 100 \mathrm{~g}^{-1}$ en 14 genotipos de Avena sativa y en el rango de los valores reportados por Kafilzadeh y Heidary (2013) que variaron de 5.2 a $12.2 \mathrm{~g} 100$ $\mathrm{g}^{-1}$ en 18 variedades de Avena sativa cosechadas en el estado de grano masoso. En los primeros cortes, la PC en planta completa no fue tan alta como en hoja y en los últimos cortes no fue tan alta como en panícula. Esto se puede deber a que de los 90 a los 150 días de edad, en hojas disminuyó mucho la PC y en panícula no disminuyó.

En hoja y tallo, los contenidos de FDN, FDA, LC y GC aumentaron al transcurrir el tiempo, mientras que los de PC y Cen disminuyeron. Excluyendo GC, el cambio de estos contenidos en maleza fue similar a los de hoja y tallo. En panícula, los contenidos de FDN y FDA disminuyeron, mientras que los de Cen,
LC, PC y GC aumentaron. Los resultados en planta completa fueron similares a los de hoja, tallo, maleza y panícula. Al respecto El-Shatnawi et al. (2004) encontraron que el valor de fibra cruda aumentó $35 \%$ de

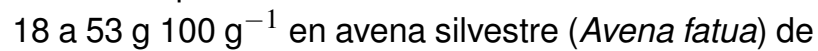
60 a 140 días después de la emergencia.

Los contenidos de PC y LC de este estudio, en planta completa a 150 días de edad cayeron en el rango de los valores reportados por Kafilzadeh y Heidary (2013) para 19 variedades de Avena sativa (PC: 5.2 a $12.2 \%$, LC: 3.31 a $5.8 \%$ ) y los contenidos de FDN y FDA obtenidos en este estudio fueron levemente superiores a los valores máximos obtenidos por estos investigadores (FDN: 53.1 a 59.7\%, FDA: 30.6 a 39.9\%). Al respecto Ortiz-Tirado et al. (2019) encontraron en forraje hidropónico de

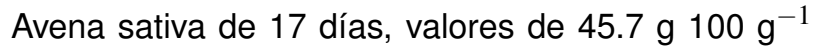

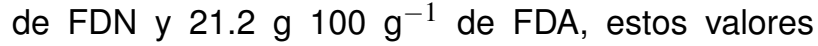
fueron similares a los obtenidos en el presente trabajo a los 45 días de edad (Tabla 5). Los valores de GC en planta completa a los 150 días de edad de este estudio, fueron inferiores, y los de cenizas fueron superiores a los reportados por Harikesh et al. (2017) en el segundo corte de Avena sativa $(1.78 \mathrm{~g}$

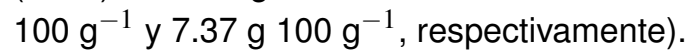

\section{Rendimientos de nutrientes}

En hoja, el máximo RPC (401 kg ha $\left.{ }^{-1}\right)$ ocurrió a los 75 días de edad. En tallo, el máximo RPC (147 $\mathrm{kg} \mathrm{ha}^{-1}$ ) ocurrió a los 90 días, pero el rendimiento a los 75 días fue de $134 \mathrm{~kg} \mathrm{ha}^{-1}$. En maleza el rendimiento máximo (52 kg ha ${ }^{-1}$ ) ocurrió a los 75 días de edad. En planta completa el RPC máximo $\left(587 \mathrm{~kg} \mathrm{ha}^{-1}=401+134+52 \mathrm{~kg} \mathrm{ha}^{-1}\right)$ ocurrió a los 75 días. A esta edad no se detectaron cantidades considerables de panícula, por lo que el RPC de este componente se consideró cero. Esto significa que a esta edad tiene más peso la hoja que los demás componentes sobre el RPC de la planta completa. En este trabajo, el RPC a los 150 días de edad en planta completa fue de $368 \mathrm{~kg} \mathrm{ha}^{-1}$. Todos los RPC (Figura 5) cayeron en el rango de 253 a $707 \mathrm{~kg}$ $\mathrm{ha}^{-1}$, reportado para 14 genotipos de Avena sativa (Sahu y Tiwari 2020). En hoja, los RFDN y RFDA fueron máximos a los 75 días de edad. Contraria- 
mente, en tallo a los 75 y 90 días de edad los RFDN y RFDA no fueron máximos, estos rendimientos fueron máximos en tallo, después de los 135 días de edad. En panícula, ningún rendimiento fue máximo a los 75 días de edad.

\section{CONCLUSIONES}

Para que los contenidos y rendimientos de pro- teína cruda no sean tan bajos, se sugiere cosechar el forraje de avena entre los 75 y 90 días de edad. Por ejemplo, a los 90 días de edad, los contenidos de pro-

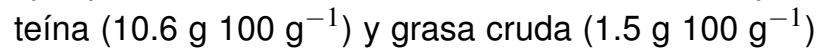
no son tan bajos, los de fibra detergente neutro (59.6 $\mathrm{g} 100 \mathrm{~g}^{-1}$ ), no son tan elevados y los rendimientos de materia seca y proteína cruda (5030 y $450.2 \mathrm{~kg}$ $\mathrm{ha}^{-1}$ ) son cercanos a sus respectivos máximos (5693 y $587.3 \mathrm{~kg} \mathrm{ha}^{-1}$ ).

\section{LITERATURA CITADA}

AOAC (1980) Methods of analysis of the Association of Official Analytical Chemists. 15th (Ed.). Arlington. VA: Association of Official Analytical Chemists.USA. 771p.

Dhakre DS, Bhattacharya D, Nath B (2018) SPSS Practical manual on randomized block design (RBD). Institute of Agriculture, Visva-Bharati, Sriniketan, West Bengal-731 236, India. 8 p. http://psbvb.in/rbd.pdf. Fecha de consulta: noviembre de 2021.

El-Shatnawi MKJ, Saoub HM, Haddad NI (2004) Growth and chemical composition of wild oat (Avena fatua) under Mediterranean conditions. Grass and Forage Science 59: 100-103.

Espitia RE, Villaseñor MHE, Tovar GR, de la O OM, Limón OA (2012) Momento óptimo de corte para rendimiento y calidad de variedades de avena forrajera. Revista Mexicana de Ciencias Agrícolas 3: 771-783.

García E (2004) Modificaciones al Sistema de Clasificación Climática de Köppen. $4^{a}$ Ed. Universidad Nacional Autónoma de México. México. 217p.

Giraldo LA, Gutiérrez LA, Rúa C (2007) Comparación de dos técnicas in vitro e in situ para estimar la digestibilidad verdadera en varios forrajes tropicales. Revista Colombiana de Ciencias Pecuarias 20: 269-279.

González SS (1993) Improving utilization of poor-quality forages with yeast culture. In: Lyons TP (ed). Biotechnology in the feed industry. Proceedings of the Ninth Annual Symposium. Nicholasville, Kentucky, USA. pp: 255-267.

Harikesh J, Kaushik MK, Choudhary JL (2017) Agronomic evaluation of oat (Avena sativa L.) for growth, yield and quality with varying levels of irrigation and nitrogen. Annals of Plant and Soil Research 19: 385-388.

Hernández-Garay A, Martínez HPA (1997) Utilización de pasturas tropicales. En: Torres HG, Díaz RP (ed). Producción de ovinos en zonas tropicales. Fundación produce-INIFAP. México. pp: 8-24.

Kafilzadeh F, Heidary N (2013) Chemical composition, in vitro digestibility and kinetics of fermentation of wholecrop forage from 18 different varieties of oat (Avena sativa L.). Journal of Applied Animal Research 41: 61-68.

Leyva-Mir SG, Villaseñor-Mir HE, Camacho-Tapia M, Ávila-Quezada GD, García-León E, Tovar-Pedraza JM (2018) Respuesta de genotipos de avena a la infección por Puccinia graminis f. sp. avenae en Valles Altos de México. Revista Mexicana de Ciencias Agrícolas 9: 317-328.

Navarro-Ortiz CA, Roa-Vega ML (2018) Comparación de la digestibilidad de tres especies forrajeras estimada mediante diferentes técnicas. Orinoquia 22: 15-33. 
Ortiz-Tirado P, Paillacho N, Barros-Rodríguez M, Mayorga-Paredes S, Chay-Canul A, Guishca-Cunuhay C, Romero-Herrera M, Reyes H (2019) Influence of cutting age on chemical composition, rumen degradation kinetics and in vitro digestibility of green hydroponic fodder of Avena sativa. Tropical and Subtropical Agroecosystems 22: 819-825.

Sahu M, Tiwari A (2020) Genetic variability and association analysis of oat (Avena sativa L.) genotypes for green forage yield and other components. Current Journal of Applied Science and Technology 39: 133-141.

SIAP (2018) Servicio de información Agroalimentaria y Pesquera. Avance de siembras y cosechas. Resumen por cultivo. http://infosiap.siap.gob.mx:8080/agricola_siap_gobmx/ResumenDelegacion.do. Fecha de consulta: octubre de 2020.

Van Soest P (1994) Nutritional ecology of the ruminant. 2nd Ed. Cornell University Press. Ithaca, New York. $478 p$. 